\title{
DNA Vector pPRA-PSM Vaccine
}

National Cancer Institute

\section{Source}

National Cancer Institute. DNA Vector PPRA-PSM Vaccine. NCI Thesaurus. Code C67089.

A cancer vaccine consisting of a DNA plasmid encoding epitopes of the human preferential antigen of melanoma (PRAME) and the prostate specific membrane antigen (PSMA) with potential immunostimulating activity. Upon direct administration of this vaccine into lymph nodes, peptides expressed by DNA plasmid vector pPRA-PSM may activate the immune system, resulting in a cytotoxic T-lymphocyte (CTL) response against PRAME- and PSMA-expressing cells. PRAME and PSMA are tumor associated antigens upregulated in a number of cancer cell types. As part of the MKC1106-PP regimen exploiting the 'prime-boost strategy', this plasmid is responsible for priming the immune response and is used in conjunction with a peptide vaccine consisting of PRAME and PSMA that boosts the immune system against PRAME- and PSMA-expressing tumor cells. 